\title{
Fusões e aquisições no ensino superior à luz da teoria do crescimento da firma
}

\author{
Edson Andrade dos Doutorando em Administração. Universidade do Vale do Itajaí (UNIVALI) - \\ Reis Brasil. edsonreisdr@gmail.com \\ Raul Beal Partyka Mestrando em Administração. Universidade do Vale do Itajaí (UNIVALI) - Brasil. \\ raul@edu.univali.br \\ Jeferson Lana Doutor em Administração. Universidade do Vale do Itajaí (UNIVALI) - Brasil. \\ jlana@univali.br \\ Rosilene Marcon Doutora em Engenharia da Produção. Universidade do Vale do Itajaí (UNIVALI) \\ - Brasil.marcon@univali.br
}

\section{RESUMO}

Este artigo analisa 18 anos (2000-2017) de publicações sobre Fusões e Aquisições de Instituições de Ensino Superior ao redor do mundo. O levantamento bibliométrico encontrou cento e oitenta e sete artigos cujo foco foi a intersecção destes temas. Aplicados os devidos filtros, a amostra final contou com cinquenta e um artigos. Dentre os principais achados, obtidos com análise destes cinquenta e um artigos, observa-se que há predominância de pesquisas qualitativas e sem a ocorrência de publicação cuja base teórica baseia-se na Teoria do Crescimento da Firma. Grande parte dos artigos encontrados têm sua origem em vertentes oriundas da economia e educação. A ausência de pesquisas sobre Fusões e Aquisições no subsetor de ensino superior revela uma excelente contribuição deste artigo, pois traz um prisma inexplorado e relevante a este tipo de fenômeno. Nos últimos dez anos, apenas no Brasil, mais de duas centenas de fusões e aquisições de instituições de Ensino Superior ocorreram. A contribuição teórica e empírica pretendida com os resultados aqui apresentados é impulsionar futuras pesquisas sobre F\&A no setor do ensino superior à luz da Teoria do Crescimento da Firma, de Penrose (1959). Além disso, este artigo também visa demonstrar a importância da produção de artigos quantitativos para testar hipóteses relacionadas às fusões e aquisições, principalmente estudos que possam analisar amostras significativas dentro do universo nacional e que utilizem variáveis outras que não apenas aquelas relacionadas às performances financeiras.

Palavras-chave: Teoria do Crescimento da Firma. Fusões e Aquisições. Ensino Superior.

\section{Mergers and acquisitions in higher education based on the theory of the growth of the firm}

\begin{abstract}
This article analyzes 18 years (2000-2017) of publications on Mergers and Acquisitions of Higher Education Institutions around the world. The bibliometric survey found one hundred and eighty-seven articles whose focus was the intersection of these themes. Once the filters were applied, the final sample had fifty-two articles. Among the main findings obtained with the analysis of these fifty-two articles, it is observed that there is a predominance of qualitative researches and without the occurrence of publication whose theoretical basis is based on the Theory of Firm Growth. Most of the articles found have their roots in economics and education. The lack of research on Mergers and Acquisitions in the subsector of higher education reveals an excellent contribution of this article, since it brings an unexplored and relevant prism to this type of phenomenon. In the
\end{abstract}


last ten years, in Brazil alone, more than two hundred mergers and acquisitions of higher education institutions occurred. The expected theoretical and empirical contribution to the results presented here is to foster future research on $\mathrm{M} \& \mathrm{E}$ in the higher education sector in light of Penrose's Theory of Firm Growth (1959). In addition, this article also aims to demonstrate the importance of the production of quantitative articles to test hypotheses related to mergers and acquisitions, mainly studies that can analyze significant samples within the national universe and that use variables other than those related to financial performance.

Keywords: Theory of the growth of the firm. Mergers and acquisitions. Higher education. 1 INTRODUÇÃO

Um dos papéis fundamentais de um gestor é maximizar a riqueza dos acionistas, seja maximizando o valor corrente das ações unitárias ou mesmo criando estratégias que beneficiam a expansão do negócio alinhado ao propósito do acionista (JENSEN; MECKLING, 1976). Fusões e aquisições (F\&A) têm sido consideradas como excelentes mecanismos de expansão da firma. Nas F\&A, espera-se algum ganho em ambas as empresas participantes do processo. Alguns dos motivos podem ser o aumento de participação de mercado, ganhos de escala, possibilidades de entrada em novos mercados, bem como outros motivos diversos.

A linha de estudo de F\&A ainda não demostrou solidez suficiente para que se torne uma teoria com todos os seus pressupostos necessários. Assim, diversos autores que estudam o tema de $F \& A$, embasam suas pesquisas em teorias mais consolidadas, como a Teoria da Firma (COASE, 1937), a Teoria do Crescimento da Firma (PENROSE, 1959) e a Teoria da Agência (JENSEN; MECKLING, 1976), por exemplo.

Na teoria do crescimento da firma de Penrose (1959), exploram-se os aspectos ligados ao crescimento da firma, mas não necessariamente ao tamanho dela. Para Penrose (1959), o tamanho da firma é apenas um subproduto do processo de crescimento e que, assim, não existe um tamanho ótimo, ou mesmo mais rentável. Esta abordagem, aponta para um diferente foco de análise quando comparado aos estudos em que economistas tratam do tamanho da firma.

Neste sentido, uma teoria do crescimento da firma deve explicar vários tipos de crescimento qualitativamente diferentes e deve levar em conta não só a sequência de mudanças geradas pelas próprias atividades da empresa, mas também o efeito de mudanças externas à empresa e que estão além do seu controle (PENROSE, 1959).

Antes de discorrer sobre a expansão da firma pelas F\&A, Penrose (1959) apresenta três classes limitadoras do crescimento, as quais são: a capacidade gerencial; fatores de mercado ou produtos e incerteza e risco. A capacidade gerencial, refere-se a condições internas da empresa, já os fatores de mercado ou produtos a condições externas. Com relação incerteza e risco, forma-se uma combinação de atitudes internas e condições externas.

No que diz respeito à expansão por meio das F\&A, Penrose (1959) destaca que para a aquisição ocorrer, deve existir um vendedor, bem como um comprador, ambos com expectativas de ganho na transação. Entretanto, ressalta que algumas vezes o vendedor está sob pressão do comprador, o que invalida esta afirmação, mesmo que ela seja verdadeira.

As F\&A como mecanismo de expansão apresentam tanto vantagens quanto limitações. Uma das vantagens propostas por Penrose (1959), é o fato de adquirir a empresa por um preço menor que o seu custo de produção, o que resulta em ganhos advindos da compra em razão do preço, bem como o posicionamento de mercado pós-aquisição. No mais, a capacidade técnica da empresa adquirida torna-se um diferencial para a compradora, fazendo com que o ingresso em novos setores seja facilitado. Já com relação às limitações, a adaptação da empresa frente às políticas do novo grupo é uma delas, sejam elas adaptações financeiras ou de recursos humanos.

Neste contexto, os mais diversos setores têm-se utilizado das F\&A como mecanismo de expansão. A JBS, nos últimos anos adquiriu empresas do setor alimentício, expandindo sua participação no mercado mundial e se tornando um grupo entre os maiores do setor. A América Latina Logística e a Comgás foram adquiridas pela Cosan. Já a Azul e a Trip do setor de aviação brasileiro, se fundiram. Inúmeras são as F\&A realizadas constantemente. 
A expansão do setor educacional no país é convergente com o desenvolvimento econômico, político e social. Empresas das mais diversas áreas têm demandado cada vez mais recursos humanos advindos da educação superior e isso faz com que empresas em busca da maximização da riqueza dos acionistas se expandam, inclusive por meio das F\&A, dando assim, maior aceitação no uso da teoria do crescimento da firma.

Diante destas questões, o cenário brasileiro ainda se mantém como uma incógnita com relação aos estudos acerca das F\&A no setor educacional. Assim, este estudo tem a pretensão de verificar qual a lacuna de investigações de F\&A no Ensino Superior à luz da Teoria do Crescimento da Firma de Penrose, e entender como a comunidade acadêmica tem se posicionado, tanto nacional quanto internacionalmente. Neste sentido, uma pesquisa bibliométrica foi efetuada para definir o atual estado da arte das investigações acerca das F\&A no Ensino Superior. De um total aproximado de 200 artigos encontrados inicialmente, 51 artigos compuseram as análises deste estudo. Como principais achados, pode-se se citar que a grande maioria dos artigos trata das F\&A a partir do ponto de vista econômico ou educacional, sem considerar aspectos quantitativos ou teorias organizacionais.

\section{REVISÃO DA LITERATURA}

Esta revisão da literatura visa reunir as discussões das F\&As com a Teoria do Crescimento da Firma de Penrose, e também a relação das $F \& A s$ com o setor educacional brasileiro. A seção também precede a demonstração do método utilizado.

\subsection{As F\&As e a Teoria do Crescimento da Firma de Penrose}

Antes de discorrer sobre a expansão da firma pelas F\&A, Penrose (1959) apresenta três classes limitadoras do crescimento, as quais são: a capacidade gerencial; fatores de mercado ou produtos e incerteza e risco. A capacidade gerencial, refere-se a condições internas da empresa, já os fatores de mercado ou produtos a condições externas. Com relação incerteza e risco, forma-se uma combinação de atitudes internas e condições externas. Assim, é bastante razoável supor que, em qualquer momento e em qualquer economia, os montantes de mão de obra, serviços gerenciais e de capital e as oportunidades de investimento lucrativo sejam mais ou menos fixos e não facilmente expansíveis, embora possam aumentar ao longo do tempo (PENROSE, 1959).

Nesta perspectiva, a expansão não se dá automaticamente, mas depende de sua capacidade gerencial, por exemplo. Assim, a expansão precisa ser programada, bem como sua execução precisa de planejamento. Para isso, é ideal que os recursos necessários para a expansão estejam disponíveis para a empresa. Penrose (1959) discorre que é obvio que se todos os recursos estivessem disponíveis de forma ilimitada, nem um plano seria ideal, pois o melhor plano seria aquele com expansão contínua, ou seja, o maior plano seria sempre melhor em detrimento de um menor. Desta maneira, deve haver alguma limitação com relação ao plano. Ainda relacionado à questão da capacidade gerencial, Penrose (1959) relata que se o argumento aceito é de que uma empresa se expandirá apenas de acordo com planos de expansão e que a extensão desses planos será limitada pelo tamanho do grupo gestor, então é evidente que, conforme os planos são concluídos e colocados em operação, os serviços gerenciais absorvidos nos processos de planejamento serão liberados gradualmente e estarão disponíveis para planejamento adicional (PENROSE, 1959).

A incerteza e o risco como limitadores do crescimento estão, por sua vez, relacionados a questões futuras, e por isso não são facilmente expostos. Na Teoria do Crescimento da Firma, Penrose (1959), descreve que a incerteza se refere à confiança do empreendedor em suas estimativas ou expectativas, já o risco, por outro lado, refere-se aos possíveis resultados da ação, especificamente para a perda que poderia ser incorrida se uma determinada ação é tomada. Neste sentido, surge a questão acerca da incerteza e risco. De forma simples, empreendedores projetam os cenários de expansão usando estimativas mais baixas para as receitas e mais altas para os custos, por exemplo. No processo de F\&A não é diferente, assim a expansão não deve se basear apenas em perspectivas de projeção de cenários de forma a projetar as receitas a níveis mais baixos e os custos em patamares maiores, na tentativa de minimizar os respectivos riscos e incertezas. 
No processo de $F \& A$, Ross et al (2015) descrevem que as aquisições seguem uma das três formas básicas: fusão ou incorporação, aquisição de ações e aquisição de ativos. Assim, uma incorporação refere-se à absorção de uma empresa por outra. A fusão se dá da mesma forma que uma incorporação, exceto que uma empresa totalmente nova é criada. Já uma oferta de aquisição é uma oferta pública para comprar ações de uma empresa-alvo. Neste sentido, ela é feita por uma empresa diretamente aos acionistas de outra empresa. Na terceira forma, uma empresa pode adquirir outra comprando todos os seus ativos. Uma vantagem aqui é que, embora o adquirente, muitas vezes, fique com uma minoria de acionistas em uma aquisição de ações, isso não acontece em uma aquisição de ativos. Ross et al (2015), classificam ainda a aquisição em três tipos, os quais são a aquisição horizontal em que a adquirente e a adquirida são do mesmo setor, a aquisição vertical em que envolve empresas em diferentes etapas do processo produtivo e a aquisição conglomerada em que a empresa adquirente e a empresa adquirida não estão relacionadas umas à outra.

Na TCF de Penrose, o termo fusão é utilizado para designar qualquer método de combinação de empresas existentes, quer seja a aquisição de uma empresa por outra, a combinação de duas empresas em pé de igualdade ou a reorganização de toda uma indústria através de uma consolidação das empresas que a compõem. Assim, a fusão como método de expansão de uma empresa individual é apenas a aquisição de uma empresa por outra. As outras duas formas de fusão envolvem mais do que simples expansão e aqui serão chamadas de combinação e consolidação, respectivamente (PENROSE, 1959).

Cabe ressaltar que para Penrose (1959), se uma expansão planejada é considerada lucrativa, independentemente de qualquer mudança na posição existente de outros produtores ou na distribuição da propriedade de ativos industriais existentes, a empresa escolherá expandir por meio da aquisição somente se a aquisição for considerada mais barata do que a expansão interna. Assim, a aquisição só ocorrerá se existirem empresas dispostas a abandonar os seus ativos, incluindo a sua "boa vontade", conforme colocado pela autora, a um preço igual ou inferior ao seu valor para os potenciais compradores. Desta forma, para que a aquisição ocorra, deve haver um vendedor, bem como um comprador, em que ambos ganhem com a transação.

\subsection{As F\&As e o setor educacional brasileiro}

Em 2005 ocorreu um caso de grande relevância com relação as $F \& A$ entre instituições de ensino superior no Brasil. O grupo norte-americano Laureate Education, fez em dezembro de 2005 a aquisição de $51 \%$ da Universidade Anhembi-Morumbi, dando início a uma onda de F\&A no setor. Neste sentido, a consultoria Hoper, apresentou em sua pesquisa em 2014 que o setor educacional privado expôs uma receita líquida de 32 bilhões de reais em 2013, representando diretamente no PIB nominal brasileiro um percentual de $0,7 \%$.

A tabela a seguir apresenta as principais atividades de F\&A no setor de ensino superior dos últimos anos.

Tabela 1 - Principais atividades de F\&A no Ensino Superior desta década.

\begin{tabular}{|c|c|c|c|c|c|}
\hline DATA & EMPRESA ALVO & COMPRADOR & $\begin{array}{c}\text { VALOR } \\
\text { (R\$ } \\
\text { MILHÕES) }\end{array}$ & $\begin{array}{l}\text { NÚMERO } \\
\text { DE } \\
\text { ALUNOS }\end{array}$ & $\begin{array}{l}\text { VALOR/A } \\
\text { LUNO } \\
\text { (R\$) }\end{array}$ \\
\hline Março/2010 & $\begin{array}{l}\text { Grupo IUNI - } \\
\text { UNIC/UNIME/FAMA/ } \\
\text { UNIRON }\end{array}$ & $\begin{array}{l}\text { Kroton } \\
\text { Educacional }\end{array}$ & 270,00 & 42.000 & $6.428,57$ \\
\hline Setembro/2011 & UNIBAN & $\begin{array}{l}\text { Anhanguera } \\
\text { Educacional }\end{array}$ & 382,60 & 55.100 & $6.943,74$ \\
\hline Dezembro/2011 & $\begin{array}{l}\text { Universidade Norte } \\
\text { do Paraná (Unopar) }\end{array}$ & $\begin{array}{l}\text { Kroton } \\
\text { Educacional }\end{array}$ & $1.300,00$ & 162.000 & $8.024,69$ \\
\hline Maio/2012 & Uniasselvi & $\begin{array}{l}\text { Kroton } \\
\text { Educacional }\end{array}$ & 510,00 & 86.200 & $5 \cdot 916,47$ \\
\hline
\end{tabular}




\begin{tabular}{|c|c|c|c|c|c|}
\hline Agosto/2013 & $\begin{array}{l}\text { Centro Universitário } \\
\text { das Faculdades } \\
\text { Metropolitanas }\end{array}$ & $\begin{array}{l}\text { Lauriete } \\
\text { Internacional } \\
\text { Universities }\end{array}$ & $1.000,00$ & 66.667 & $15.000,00$ \\
\hline Agosto/2013 & $\begin{array}{ll}\text { UNISEB } & \text { Centro } \\
\text { Universitário } & \end{array}$ & $\begin{array}{l}\text { Estácio } \\
\text { Participações }\end{array}$ & 615,32 & 37.800 & $16.278,26$ \\
\hline Maio/2014 & $\begin{array}{l}\text { Anhanguera } \\
\text { Educacional } \\
\text { Participações }\end{array}$ & $\begin{array}{l}\text { Kroton } \\
\text { Educacional }\end{array}$ & $5.000,00$ & 440.000 & $11.363,64$ \\
\hline
\end{tabular}

Fonte: Adaptado de Hoper (2014).

Ao considerar a criação de valor de uma fusão, semelhanças das organizações são as principais considerações para o sucesso potencial. Supõe-se que as sinergias através do aumento geral das economias de escala e da redução de custos resultem em aumento do valor (BOLING; MAYO; HELMS, 2017). Para os autores, as $F \& A$ no ensino superior como uma alternativa estratégica para o crescimento e redução de custos continuam a aumentar em todo o mundo. Boling, Mayo e Helms (2017) destacam ainda que as tendências atuais apoiam as F\&A como uma alternativa estratégica viável para as organizações de ensino superior que buscam melhores serviços e maior valor. Assim, os processos de F\&As constituem uma maneira rápida de uma firma crescer ou internacionalizar-se, entrar em novos mercados, comprar ativos por preços favoráveis, melhorar sua base gerencial e tecnológica, aumentar a liquidez dos proprietários, elaborar estratégia de defesa contra aquisições hostis, buscar sinergias, ou oportunidades de alguns participantes lucrarem em detrimento de outros (CAMARGOS; COUTINHO 2008).

Ainda que os principais estudos acerca das F\&A discorrem sobre as determinantes da fusão, é importante ressaltar as métricas avaliativas pós-fusão. Os estudos propostos no ensino superior, percorrem os mais diversos campos de análise. A proporção entre os custos dos departamentos de uma universidade comparado a quantidade de alunos, mostrará, por exemplo, se a F\&A trouxe economia em escala e redução de custos. A análise do desempenho após o processo de F\&A, também é importante, inclusive, associando a esta métrica, a quantidade de alunos do grupo.

Patterson (2000), buscou investigar 16 casos buscando evidenciar os pressupostos econômicos da economia de escala das F\&A. Para o autor, alguns estudos apontavam para economias de escopo modestas, particularmente apresentadas em grandes universidades, e várias deram ênfase à importância dos perfis institucionais e à variação de custos por aluno. Economias de escala entre cursos de graduação e pós-graduação também foram encontradas. Em uma outra perspectiva, Harman (2002a), pesquisou a integração cultural pósfusão. O autor descreve que em qualquer processo de rápida mudança organizacional, os afetados tendem a se sentir desorientados, perturbados, frustrados, despreparados para a mudança e incapazes de competir com as demandas da instituição recém-criada. Assim, percebe-se que o processo de F\&A no ensino superior não traz resultados apenas econômicos. Dado que a criação de valor ao acionista é resultado de políticas de longo prazo, questões culturais de integração e mudança organizacional devem ser levadas em consideração.

Em uma mesma perspectiva, Hay e Fourie (2002) analisam a percepção dos funcionários com relação a F\&A e os resultados indicam que o pessoal não se opõe à ideia da $F \& A$, entretanto, deve ser dada atenção cuidadosa aos fatores particularmente pessoais e que os receios do pessoal terão de ser abordados no processo para garantir uma fusão eficaz.

Wan e Peterson (2007), examinam o planejamento antes da F\&A, o processo de integração pós $F \& A$ e os resultados da fusão. Assim, descrevem que a decisão de uma aquisição ou fusão, vem de cima para baixo e que uma das questões mais importantes identificadas na pesquisa é a dificuldade de construir uma identidade unificada para a nova instituição.

Tabela 2 - Evolução de receita líquida dos principais players da educação superior brasileira.

\begin{tabular}{cccccccc}
\hline $\begin{array}{c}\text { RANKING } \\
2013\end{array}$ & EDUCACIONAL & 2008 & 2010 & 2011 & 2012 & 2013 & $\begin{array}{c}\text { CRESCIMENTO ANUAL } \\
\text { COMPOSTA (2008- } \\
\text { 2013) }\end{array}$ \\
\hline
\end{tabular}




\begin{tabular}{|c|c|c|c|c|c|c|c|}
\hline 1 & $\begin{array}{l}\text { Kroton } \\
\text { Educacional }\end{array}$ & 280 & 642 & 1115 & 1405 & 2015 & $48,4 \%$ \\
\hline 2 & Anhanguera & 654 & 1004 & 1213 & 1607 & 1812 & $22,6 \%$ \\
\hline 3 & Estácio & 980 & 1016 & 1148 & 1383 & 1731 & $12,1 \%$ \\
\hline 4 & $\begin{array}{l}\text { UNIP + Holding Di } \\
\text { Gênio }\end{array}$ & 1012 & 110 & 1430 & 1376 & 1431 & $7,2 \%$ \\
\hline 5 & $\begin{array}{l}\text { Lauriete } \\
\text { International }\end{array}$ & 425 & 763 & 702 & 956 & 1115 & $21,3 \%$ \\
\hline 6 & Grupo UNINOVE & 366 & 487 & 520 & 562 & 584 & $9,8 \%$ \\
\hline 7 & Grupo UNICSUL & 276 & 228 & 340 & 487 & 529 & $13,9 \%$ \\
\hline 8 & Ànima Educação & 254 & 264 & 265 & 400 & 461 & $12,7 \%$ \\
\hline 9 & Ser Educacional & 115 & 160 & 172 & 282 & 457 & $31,8 \%$ \\
\hline 10 & Whitney & - & 232 & 239 & 312 & 343 & $13,9 \%$ \\
\hline 11 & DeVry & 80 & 104 & 120 & 180 & 242 & $24,8 \mid \%$ \\
\hline 12 & Grupo Tiradentes & - & 126 & 136 & 197 & 236 & $23,3 \%$ \\
\hline \multicolumn{2}{|c|}{ TOTAL DO SETOR } & 24100 & 28200 & 29200 & 28230 & 32040 & $5,9 \%$ \\
\hline
\end{tabular}

Fonte: Adaptado de Hoper (2014).

Diante de diversos achados, conforme os da Tabela 2, é importante levar em consideração o crescimento do setor educacional. Hoper (2014) apresenta a evolução da receita líquida dos principais grupos da educação superior brasileira, o que demonstra o crescimento anual entre os anos de 2008 e 2013.

\section{METODOLOGIA}

A partir de um levantamento bibliométrico, este estudo buscou analisar os principais artigos publicados internacionalmente e nacionalmente, enquadrados dentro da área de fusões e aquisições no ensino superior.

Para analisar a produção científica com o tema fusões e aquisições no ensino superior no período de 2000 a 2017, foram coletadas as produções correlatas nas seguintes bases: Scopus e Web of Science. Os principais filtros aplicados à pesquisa foram: i) definidas as métricas relacionadas às expressões e palavras de interesse, a saber Higher Education "AND" Merger "OR" Acquisition; ii) aplicados os filtros temporais, com período de pesquisa maior que 1999; iii) não foi feita distinção da área do periódico, uma vez que periódicos de educação e economia também apresentam diversos casos de pesquisas de F\&A em instituições de Ensino Superior.

Realizou-se uma busca nas bases Scopus e Web of Science, as quais mostraram apenas artigos internacionais, demonstrando a necessidade de maiores estudos no Brasil com relação a Teoria do Crescimento da Firma. Ao total, foram encontrados 116 artigos a partir da base Scopus e 187 artigos pela base Web of Science. Por apresentar um número mais elevado, efetuou-se o download dos 187 artigos da Web of Science. O próximo passo da pesquisa foi a avaliação individual dos artigos a partir do título, resumo e considerações finais, para certificar que a base final contivesse apenas artigos cujo foco fosse na investigação do fenômeno das F\&A das instituições de Ensino Superior. Após a leitura individual das partes selecionadas dos artigos, verificou-se que 135 artigos citavam F\&A e instituições de ensino superior por outros motivos que não aqueles que classificariam a pesquisa de acordo com os filtros aqui estipulados.

A tabela 3 apresenta a quantidade de artigos analisados classificados por ano. Observa-se uma maior concentração a partir do ano de 2013. O total da amostra é de 51 artigos.

Tabela 3-Base de artigos por ano

\begin{tabular}{cccc}
\hline ANO & $\begin{array}{c}\text { QUANTIDADE DE } \\
\text { ARTIGOS }\end{array}$ & \% POR ANO & $\begin{array}{c}\% \\
\text { ACUMULADA }\end{array}$ \\
\hline 2000 & 2 & $3.85 \%$ & $3.85 \%$ \\
2001 & 0 & $0.00 \%$ & $3.85 \%$ \\
2002 & 4 & $7.69 \%$ & $11.54 \%$ \\
2003 & 2 & $3.85 \%$ & $15.38 \%$ \\
2004 & 1 & $1.92 \%$ & $17.31 \%$
\end{tabular}




\begin{tabular}{rrrr}
2005 & 2 & $3.85 \%$ & $21.15 \%$ \\
2006 & 1 & $1.92 \%$ & $23.08 \%$ \\
2007 & 3 & $5.77 \%$ & $28.85 \%$ \\
2008 & 3 & $5.77 \%$ & $34.62 \%$ \\
2009 & 1 & $1.92 \%$ & $36.54 \%$ \\
2010 & 1 & $1.92 \%$ & $38.46 \%$ \\
2011 & 2 & $3.85 \%$ & $42.31 \%$ \\
2012 & 0 & $0.00 \%$ & $42.31 \%$ \\
2013 & 6 & $11.54 \%$ & $53.85 \%$ \\
2014 & 6 & $13.46 \%$ & $67.31 \%$ \\
2015 & 7 & $13.46 \%$ & $80.77 \%$ \\
2016 & 9 & $17.31 \%$ & $98.08 \%$ \\
$2017^{*}$ & 1 & $1.92 \%$ & $100.00 \%$ \\
\hline TOTAL & $\mathbf{5 1}$ & \multicolumn{3}{c}{$\mathbf{1 0 0}$} \\
\hline
\end{tabular}

Fonte: Elaborado pelos autores com base nos dados primários. Nota: *Até o mês de março de 2017.

Destes 51 artigos finais que compuseram a base para elaboração do estudo, foram analisados, por meio de leitura integral, aspectos como: teoria utilizada, metodologia, país da amostra, journal de publicação, implicações e considerações do estudo, limitação e sugestões de futuras pesquisas.

\section{RESULTADOS E ANÁLISE}

A seguir apresentamos e discutimos os resultados sobre as teorias, as variáveis e as metodologias dos artigos.

\subsection{Principais teorias}

Inicialmente os artigos da amostra foram analisados quanto à teoria utilizada como referência. Observa-se que não há uma padronização de teorias utilizadas entre os pesquisadores. Sobretudo, e de particular interesse para este estudo, os artigos da amostra sequer citam a Teoria do Crescimento da Firma como referência principal da obra. Tais evidências que vão ao encontro, ainda que de forma marginal, do objetivo deste trabalho. A Tabela 4 apresenta as aparições das teorias como referência nas publicações pesquisadas.

Tabela 4-Teorias principais dos artigos analisados

\begin{tabular}{lcc}
\multicolumn{1}{c}{ TEORIA } & $\begin{array}{c}\text { QUANTIDADE } \\
\text { DE } \\
\text { APARIÇÕES }\end{array}$ & \\
\hline Teorias Econômicas de Crescimento & 5 & 9 \\
Economias (Escala, escopo e conhecimento) & 5 & 9 \\
Vantagem competitiva & 4 & 8 \\
Políticas (valor crítico, educacional, do ensino superior e & 4 & 8 \\
públicas) & & \\
Mudanças organizacionais e institucionais & 4 & 8 \\
Governança e sistemas de governação & 4 & 8 \\
Cultura organizacional e institucional & 4 & 8 \\
Liderança & 3 & 6 \\
Qualidade & 3 & 6 \\
Teoria da Identidade Social & 3 & 6 \\
Competitividade & 2 & 4 \\
Fusões institucionais & 2 & 4 \\
Inovação & 2 & 4
\end{tabular}




\begin{tabular}{lcc} 
Nova Gestão Pública & 2 & 4 \\
Teoria da Autocategorização & 2 & 4 \\
Outras & 80 & 150,94 \\
\hline TOTAL & $\mathbf{1 2 9}$ & \\
\hline
\end{tabular}

Fonte: Elaborado pelos autores com base nos dados primários.

Nota: Não é uma porcentagem acumulada.

\subsection{Principais Métodos}

Com relação à metodologia utilizou-se como referência quatro categorias: pesquisa qualitativa, pesquisa quantitativa, métodos mistos e ensaio teórico. O método mais utilizado foi a pesquisa Qualitativa com $52,94 \%$.

Tabela 5 - Metodologia Utilizada

\begin{tabular}{lcc}
\hline \multicolumn{1}{c}{ MÉTODOS } & $\begin{array}{c}\text { QUANTIDADE DE } \\
\text { ARTIGOS }\end{array}$ & $\%$ \\
\hline Qualitativo & 27 & 52,94 \\
Quantitativo & 18 & 35,29 \\
Misto & 2 & 3,92 \\
Ensaio Teórico & 4 & 7,84 \\
\hline TOTAL & $\mathbf{5 1}$ & $\mathbf{1 0 0}$ \\
\hline
\end{tabular}

\subsection{Principais Variáveis}

Foram compiladas na Tabela 6 as principais variáveis encontradas nos artigos analisados. Das quais a maior ocorrência foi das variáveis com Indicadores de performance operacional (24,53\%), as quais estão ligadas à melhora da qualidade do ensino e a variação do número de alunos. Ressalta-se que dos 51 artigos, 26 apresentaram variáveis em seu estudo (50,98\%).

Tabela 6 - Principais Variáveis encontradas nos artigos analisados

\begin{tabular}{lcc}
\hline \multicolumn{1}{c}{ VARIÁVEIS } & $\begin{array}{c}\text { QUANTIDADE } \\
\text { DE ARTIGOS }\end{array}$ & $\%$ \\
\hline Indicadores de performance operacional (exemplo: melhoria de & 13 & $\mathbf{2 5 , 4 9}$ \\
$\begin{array}{l}\text { qualidade de ensino, número de alunos etc.) } \\
\text { Indicadores de conhecimento (transferência de conhecimento etc.) }\end{array}$ & 7 & $\mathbf{1 3 , 7 3}$ \\
$\begin{array}{l}\text { Indicadores de performance financeira (lucro, faturamento etc.) } \\
\text { Fatores determinantes da fusão/aquisição (quando a fusão é a }\end{array}$ & 3 & 58 \\
variável dependente) & 3 & 58 \\
\hline TOTAL & & $\mathbf{2 6}$ \\
\hline
\end{tabular}

Fonte: Elaborado pelos autores com base nos dados primários.

\subsection{Principais Achados}

Com base na amostra de artigos analisados e listados no Quadro 1, as publicações na sua maioria têm base teórica em Teorias Econômicas de Crescimento, Economias (Escala, escopo e conhecimento), Vantagem competitiva, Teorias Políticas (valor crítico, educacional, do ensino superior e públicas), Mudanças organizacionais e institucionais, Governança e sistemas de governação e Cultura organizacional e institucional. 
Quadro 1 - Principais achados da amostra analisada

\begin{tabular}{|c|c|c|c|}
\hline AUTOR(ES) & ANO & JOURNAL & PRINCIPAIS ACHADOS \\
\hline Adedoyin et al. & 2015 & $\begin{array}{l}\text { Journal of Evidence- } \\
\text { Informed Social } \\
\text { Work }\end{array}$ & $\begin{array}{l}\text { Três principais fatores são fortemente sugeridos: } \\
\text { comunicação aberta e honesta, a construção de confiança, } \\
\text { e o desenvolvimento de uma visão partilhada. }\end{array}$ \\
\hline Barnard e Merwe & 2016 & $\begin{array}{l}\text { International Journal } \\
\text { of Sustainability in } \\
\text { Higher Education }\end{array}$ & $\begin{array}{l}\text { Inovação, liderança, planejamento, flexibilidade, pesquisa } \\
\text { do clima da cultura, acompanhamento dos progressos e } \\
\text { agilidade estratégica são essenciais para promover a } \\
\text { inovação entre toda a força de trabalho. }\end{array}$ \\
\hline Barrier & $2014 a$ & $\begin{array}{l}\text { Research in the } \\
\text { Sociology of } \\
\text { Organizations }\end{array}$ & $\begin{array}{l}\text { O impacto da reorganização formal nas práticas de } \\
\text { pesquisa depende do alinhamento das condições externas e } \\
\text { intraorganizacionais. }\end{array}$ \\
\hline Bates e Santerre & 2000 & $\begin{array}{l}\text { Review of Industrial } \\
\text { Organization }\end{array}$ & $\begin{array}{l}\text { Os fechamentos de colégios privados e fusões são mais } \\
\text { prováveis quando a taxa de matrícula real diminui e há um } \\
\text { aumento real de salários em faculdades privadas. }\end{array}$ \\
\hline Becker et al. & 2004 & Higher Education & $\begin{array}{l}\text { Em qualquer fusão institucional há insegurança, no entanto, } \\
\text { o fracasso em administrar sensatamente o "lado humano" } \\
\text { das fusões pode ter um impacto negativo nas ambições } \\
\text { corporativas e prolongar a transformação. }\end{array}$ \\
\hline $\begin{array}{l}\text { Beer, Smith e } \\
\text { Jansen }\end{array}$ & 2009 & $\begin{array}{l}\text { Education As } \\
\text { Change }\end{array}$ & $\begin{array}{l}\text { O desempenho acadêmico dos alunos no campus da HBU } \\
\text { foi pobre em comparação com o dos alunos do HWU }\end{array}$ \\
\hline $\begin{array}{l}\text { Boling, Mayo e } \\
\text { Helms }\end{array}$ & 2017 & $\begin{array}{l}\text { Journal of } \\
\text { Organizational } \\
\text { Change } \\
\text { Management }\end{array}$ & Houve resultados positivos nas quatro fusões BOR / USG \\
\hline $\begin{array}{l}\text { Cartwright, } \\
\text { Tytherleigh e } \\
\text { Robertson }\end{array}$ & 2007 & $\begin{array}{l}\text { European Journal of } \\
\text { Work and } \\
\text { Organizational } \\
\text { Psychology }\end{array}$ & $\begin{array}{l}\text { O estudo confirma os benefícios potenciais de programas } \\
\text { de comunicação de fusão em larga escala e consulta e } \\
\text { envolvimento dos funcionários em todos os níveis. }\end{array}$ \\
\hline $\begin{array}{l}\text { Christensen e } \\
\text { Erno-Kjolhede }\end{array}$ & 2011 & $\begin{array}{l}\text { European Journal of } \\
\text { Engineering } \\
\text { Education }\end{array}$ & $\begin{array}{l}\text { A deriva acadêmica é uma oportunidade e uma ameaça } \\
\text { para o profissional de engenharia da educação, no entanto } \\
\text { os entrevistados afirmam que as oportunidades e benefícios } \\
\text { claramente superaram ameaças e desvantagens }\end{array}$ \\
\hline Coetzee e Mbanze & 2014 & $\begin{array}{l}\text { Journal of Asian and } \\
\text { African Studies }\end{array}$ & $\begin{array}{l}\text { Recomenda-se a alteração da Lei de Educação Superior de } \\
\text { 2010, incluindo regras para regular as transferências } \\
\text { internas e externas }\end{array}$ \\
\hline Deschamps e Lee & 2015 & $\begin{array}{l}\text { Journal of Studies in } \\
\text { International } \\
\text { Education }\end{array}$ & $\begin{array}{l}\text { Aquisições e fusões são cada vez mais comuns, no entanto, } \\
\text { é preciso cautela e avaliar se a educação de qualidade é um } \\
\text { motor de internacionalização ou meramente uma desculpa } \\
\text { conveniente para aumentar as receitas }\end{array}$ \\
\hline $\begin{array}{l}\text { Docampo, Egret e } \\
\text { Cram }\end{array}$ & 2015 & Scientometrics & $\begin{array}{l}\text { Fusões fazem parte de um arsenal de ferramentas que } \\
\text { podem ser adotadas para lidar com desejos para a reforma } \\
\text { e melhoria. }\end{array}$ \\
\hline $\begin{array}{l}\text { Drowley, Lewis e } \\
\text { Brooks }\end{array}$ & 2013 & $\begin{array}{l}\text { Higher Education } \\
\text { Quarterly }\end{array}$ & $\begin{array}{l}\text { O discurso do mercado competitivo pode estar } \\
\text { profundamente enraizado no setor de ensino superior } \\
\text { galês, alimentando a resistência à visão que o governo de } \\
\text { Gales tem articulado. }\end{array}$ \\
\hline Frolich et al. & 2016 & $\begin{array}{l}\text { Tertiary Education } \\
\text { and Management }\end{array}$ & $\begin{array}{l}\text { As deliberações estratégicas parecem discutir apenas as } \\
\text { opções aparentemente disponíveis de fusão, com } \\
\text { "disponibilidade", entendida principalmente como } \\
\text { proximidade geográfica. }\end{array}$ \\
\hline Glass et al. & 2006 & $\begin{array}{l}\text { Socio-Economic } \\
\text { Planning Sciences }\end{array}$ & $\begin{array}{l}\text { As fusões para garantir maior tamanho devem ser } \\
\text { encorajadas. As universidades mais especializadas e de } \\
\text { maior porte renderiam ganhos de eficiência para o ensino } \\
\text { superior do Reino Unido. }\end{array}$ \\
\hline
\end{tabular}




\begin{tabular}{|c|c|c|c|}
\hline $\begin{array}{l}\text { Gleibs, } \\
\text { Mummendey e } \\
\text { Noack }\end{array}$ & 2008 & $\begin{array}{l}\text { Journal of } \\
\text { Personality and } \\
\text { Social Psychology }\end{array}$ & $\begin{array}{l}\text { Modelos multiníveis para a mudança confirmaram que o } \\
\text { efeito preditivo da identificação de pré-fusão na } \\
\text { identificação pós-fusão para membros da organização } \\
\text { dominante se dissipa ao longo do tempo. }\end{array}$ \\
\hline Gleibs et al. & 2013 & Social Psychology & $\begin{array}{l}\text { Os membros de grupos de alto e baixo status defendem } \\
\text { diferentes padrões de fusão porque estes presumivelmente } \\
\text { atendem às suas necessidades (status-maintenance versus } \\
\text { status-enhancement) melhor. }\end{array}$ \\
\hline Hall e Symes & 2005 & $\begin{array}{l}\text { Studies in Higher } \\
\text { Education }\end{array}$ & $\begin{array}{l}\text { Incorporações e fusões foram forçadas contra os desejos da } \\
\text { maioria das instituições envolvidas e os desafios legais } \\
\text { revelaram-se inconclusivos }\end{array}$ \\
\hline Harman & $2002 b$ & Higher Education & $\begin{array}{l}\text { Campis recém-incorporados integrados em oposição às } \\
\text { estruturas federais fornecem mais espaço para a cultura de } \\
\text { maior integração pois há o desenvolvimento de coerência } \\
\text { acadêmica e lealdade. }\end{array}$ \\
\hline Harman e Harman & 2003 & $\begin{array}{l}\text { Tertiary Education } \\
\text { and Management }\end{array}$ & $\begin{array}{l}\text { As fusões têm sido muito mais comuns nos setores públicos } \\
\text { do ensino superior do que nas instituições privadas. A fusão } \\
\text { se adequa a todas as situações. }\end{array}$ \\
\hline Harman e Harman & 2008 & $\begin{array}{l}\text { Higher Education } \\
\text { Policy }\end{array}$ & $\begin{array}{l}\text { O ambiente globalmente competitivo do ensino superior } \\
\text { proporciona fortes incentivos à concorrência e também } \\
\text { incentiva os vários tipos de parcerias entre universidades e } \\
\text { faculdades. }\end{array}$ \\
\hline Harman e Meek & 2002 & Higher Education & $\begin{array}{l}\text { Para algumas instituições, a fusão pode significar que elas } \\
\text { deixam de existir. Aqueles que sentem que perderam } \\
\text { vantagem na fusão podem continuar sua oposição muito } \\
\text { depois que os acordos são formalizados. }\end{array}$ \\
\hline Hay e Fourie & 2002 & Higher Education & $\begin{array}{l}\text { Não há oposição à ideia de combinações institucionais ou } \\
\text { de fusão, mas deve-se dar atenção a fatores } \\
\text { particularmente pessoais e que os receios do pessoal terão } \\
\text { de ser abordados no processo para ser eficaz. }\end{array}$ \\
\hline $\begin{array}{l}\text { Hidalgo-Hidalgo e } \\
\text { Valera }\end{array}$ & 2016 & $\begin{array}{l}\text { The B. E. Journal of } \\
\text { Economic Analysis \& } \\
\text { Policy }\end{array}$ & $\begin{array}{l}\text { À medida que a diferença entre as reputações iniciais das } \\
\text { instituições potencialmente em fusão aumenta, o montante } \\
\text { de fundos necessários para que as fusões obtenham uma } \\
\text { excelência superior à da concorrência também aumenta. }\end{array}$ \\
\hline Johnes & 2014 & $\begin{array}{l}\text { The Manchester } \\
\text { School }\end{array}$ & $\begin{array}{l}\text { Cinco dos sete métodos sugerem que a eficiência média no } \\
\text { setor universitário inglês no período de } 1996 / 97 \text { a 2008/9 é } \\
\text { de cerca de } 75 \% \text { a } 83 \% \text {. }\end{array}$ \\
\hline Johnes and $Y u$ & 2008 & $\begin{array}{l}\text { China Economic } \\
\text { Review }\end{array}$ & $\begin{array}{l}\text { As universidades abrangentes parecem superar as } \\
\text { instituições especializadas e a diferença é significativa } \\
\text { quando são analisadas todas as variáveis mesmo as } \\
\text { relacionadas ao aluno. A política da China de fusão de } \\
\text { universidades deve ser incentivada. }\end{array}$ \\
\hline $\begin{array}{l}\text { Karodia, Shaikh e } \\
\text { Soni }\end{array}$ & 2015 & $\begin{array}{l}\text { Mediterranean } \\
\text { Journal of Social } \\
\text { Sciences }\end{array}$ & $\begin{array}{l}\text { O exercício da fusão tem contribuído para promover a } \\
\text { desigualdade em toda a cadeia de valor e os recursos } \\
\text { humanos têm sido seriamente comprometidos. }\end{array}$ \\
\hline Kyvik & 2002 & Higher Education & $\begin{array}{l}\text { O setor universitário e a faculdade estatal individual } \\
\text { adquiriram um status mais elevado. Colocá-los sob a } \\
\text { mesma lei trouxe os aproximaram ao fazer uma distinção } \\
\text { formal. }\end{array}$ \\
\hline Kyvik e Stensaker & 2013 & $\begin{array}{l}\text { Tertiary Education } \\
\text { and Management, }\end{array}$ & $\begin{array}{l}\text { A maioria das iniciativas de fusão até agora não terminou } \\
\text { em uma decisão de fusão. }\end{array}$ \\
\hline Mabokela e Wei & 2007 & $\begin{array}{l}\text { International } \\
\text { Education Journal }\end{array}$ & $\begin{array}{l}\text { A fusão ideal deve refletir um equilíbrio delicado entre } \\
\text { unidade e diversidade, com objetivos comuns para a criação } \\
\text { de uma instituição equitativo, inclusivo. }\end{array}$ \\
\hline $\begin{array}{l}\text { Mokhuba e } \\
\text { Govender }\end{array}$ & 2016 & $\begin{array}{l}\text { Cogent Business \& } \\
\text { Management }\end{array}$ & $\begin{array}{l}\text { A falta de comunicação, a falta de convergência das partes, } \\
\text { a ausência de apoio de grupos influentes, a falta de }\end{array}$ \\
\hline
\end{tabular}




\begin{tabular}{|c|c|c|c|}
\hline & & & $\begin{array}{l}\text { estratégias para lidar com a mudança e a falta de confiança } \\
\text { são os maiores desafios relacionados com a fusão. }\end{array}$ \\
\hline Ngcamu et. al. & 2015 & $\begin{array}{l}\text { Problems and } \\
\text { Perspectives in } \\
\text { Management }\end{array}$ & $\begin{array}{l}\text { Os líderes universitários não têm a capacidade pessoal de } \\
\text { aprender com seus erros. E estão mais interessados em } \\
\text { estabelecer, manter ou melhorar as relações externas ou as } \\
\text { parcerias do que as internas. }\end{array}$ \\
\hline Ngcamu & 2016 & $\begin{array}{l}\text { International Journal } \\
\text { of Sociology and } \\
\text { Social Policy }\end{array}$ & $\begin{array}{l}\text { A transformação nas instituições de ensino superior é } \\
\text { definida por fatores internos (operacionais e fundamentais) } \\
\text { e externos com influência direta. }\end{array}$ \\
\hline Ngcamu e Teferra & 2015 & $\begin{array}{l}\text { International Journal } \\
\text { of Science Education }\end{array}$ & $\begin{array}{l}\text { Os fatores internos que definem a transformação incluem o } \\
\text { acesso e a retenção dos alunos e o alcance das metas } \\
\text { estabelecidas pela universidade. }\end{array}$ \\
\hline Patterson & 2000 & $\begin{array}{l}\text { Tertiary Education } \\
\text { and Management }\end{array}$ & $\begin{array}{l}\text { Alianças são comumente propostas e engendradas em uma } \\
\text { expectativa de benefício econômico. A expectativa é de } \\
\text { economias de escala e eficiência de custos de operação. }\end{array}$ \\
\hline Pavlik & 2016 & $\begin{array}{l}\text { Public Finance } \\
\text { Quarterly }\end{array}$ & $\begin{array}{l}\text { A estrutura de propriedade, o sistema de contabilidade, a } \\
\text { ciclicidade do ensino superior, devem ser levados em conta } \\
\text { no sentido econômico-jurídico-financeiro, para as } \\
\text { necessidades sociais e a máxima proteção dos interesses } \\
\text { dos estudantes. }\end{array}$ \\
\hline Pick & 2003 & $\begin{array}{l}\text { Tertiary Education } \\
\text { and Management }\end{array}$ & $\begin{array}{l}\text { A visão de que as instituições de ensino superior são } \\
\text { principalmente formadores de cultura nacional e cidadania } \\
\text { e a visão de que as universidades devem se comportar mais } \\
\text { como empresas privadas que operam em um mercado } \\
\text { global de ensino superior são divididas e não são exclusivas } \\
\text { da Austrália regional. }\end{array}$ \\
\hline Puusa e Kekale & 2013 & $\begin{array}{l}\text { Tertiary Education } \\
\text { and Management }\end{array}$ & $\begin{array}{l}\text { Os membros da equipe se comprometem com seu trabalho } \\
\text { e, devido à mudança, também cada vez com seus colegas } \\
\text { próximos. }\end{array}$ \\
\hline Puusa e Kekale & 2015 & $\begin{array}{l}\text { Journal of Higher } \\
\text { Education Policy and } \\
\text { Management }\end{array}$ & $\begin{array}{l}\text { Pressões externas e mudanças na legislação resultam em } \\
\text { mudanças organizacionais que precisam ser realizadas pela } \\
\text { liderança. }\end{array}$ \\
\hline $\begin{array}{l}\text { Ripoll-Soler e } \\
\text { Molina }\end{array}$ & 2013 & $\begin{array}{l}\text { Revista de } \\
\text { Educación }\end{array}$ & $\begin{array}{l}\text { A fusão nem sempre resolve os problemas anteriores. } \\
\text { Alguns fatores-chave para o sucesso ou fracasso são do } \\
\text { tamanho da instituição resultante ou o desenvolvimento de } \\
\text { uma nova cultura organizacional. }\end{array}$ \\
\hline $\begin{array}{l}\text { Ripoll-Soler e } \\
\text { Molina }\end{array}$ & 2014 & $\begin{array}{l}\text { Tertiary Education } \\
\text { and Management }\end{array}$ & $\begin{array}{l}\text { É necessário um estudo aprofundado na escolha de um } \\
\text { determinado modelo de colaboração interinstitucional, } \\
\text { especialmente as fusões. }\end{array}$ \\
\hline Saarti e Juntunen & 2011 & Library Management & $\begin{array}{l}\text { A biblioteca poderia utilizar seu sistema de gerenciamento } \\
\text { de qualidade na fusão das duas bibliotecas e sua produção } \\
\text { de serviços. É importante que todo o pessoal participe do } \\
\text { processo. }\end{array}$ \\
\hline Sehoole & 2005 & Higher Education & $\begin{array}{l}\text { O documento reconhece que a maioria dos estudos } \\
\text { publicados sobre fusões se originam em economias } \\
\text { estabelecidas como as da Noruega, Austrália, Inglaterra, } \\
\text { EUA. }\end{array}$ \\
\hline $\begin{array}{l}\text { Slade, Ribando e } \\
\text { Fortner }\end{array}$ & 2016 & Scientometrics & $\begin{array}{l}\text { Professores universitários apresentam maior estresse no } \\
\text { trabalho do que membros de maior status. Este aumento } \\
\text { do estresse para os membros inferiores status de } \\
\text { universidade acaba em resultar maior tempo gasto em } \\
\text { pesquisa. }\end{array}$ \\
\hline Tight & 2013 & $\begin{array}{l}\text { Journal of Higher } \\
\text { Education Policy and } \\
\text { Management }\end{array}$ & $\begin{array}{l}\text { No período } 1994-1995 \text { a } 2009 / 10 \text {, o número de instituições } \\
\text { de ensino superior no Reino Unido caiu de } 182 \text { para } 165 . \\
\text { Consequência das fusões institucionais, mudanças de } \\
\text { nome, mudanças nas matrículas de estudantes, entre } \\
\text { outros. }\end{array}$ \\
\hline
\end{tabular}




\begin{tabular}{|c|c|c|c|}
\hline Ursin et al. & 2010 & $\begin{array}{l}\text { Tertiary Education } \\
\text { and Management }\end{array}$ & $\begin{array}{l}\text { A pouca atenção concreta às questões educacionais } \\
\text { relacionadas com as fusões é uma fraqueza significativa do } \\
\text { processo de planejamento. }\end{array}$ \\
\hline Vartiainen & 2016 & $\begin{array}{l}\text { Tertiary Education } \\
\text { and Management }\end{array}$ & $\begin{array}{l}\text { O sucesso da fusão da universidade consolidou a } \\
\text { cooperação entre as cidades de Joensuu e Kuopio, e } \\
\text { também muitas partes 'provinciais' interessadas. }\end{array}$ \\
\hline Walsh & 2014 & $\begin{array}{l}\text { Irish Educational } \\
\text { Studies }\end{array}$ & $\begin{array}{l}\text { Não foi possível afirmar o poder do centro político sobre um } \\
\text { setor universitário que tinha sido anteriormente autônomo, } \\
\text { mesmo a universidade com sua estrutura de governança } \\
\text { distinta, devido a defesa bem-sucedida do poder } \\
\text { institucional }\end{array}$ \\
\hline Wan e Peterson & 2007 & $\begin{array}{l}\text { International Journal } \\
\text { of Educational } \\
\text { Development }\end{array}$ & $\begin{array}{l}\text { O planejamento e a implementação da fusão foram em } \\
\text { grande parte um processo de cima para baixo e sujeito a } \\
\text { intervenção política. }\end{array}$ \\
\hline Ylijoki & 2014 & Minerva & $\begin{array}{l}\text { As reformas estruturais e transformações gerenciais, } \\
\text { promovem experiências de competição e polarização no } \\
\text { nível departamental e individual da academia. }\end{array}$ \\
\hline $\begin{array}{l}\text { Zeeman e } \\
\text { Benneworth }\end{array}$ & 2016 & $\begin{array}{l}\text { Tertiary Education } \\
\text { and Management }\end{array}$ & $\begin{array}{l}\text { O desenvolvimento local fica em risco devido ao crescente } \\
\text { isolamento dos campi de seus ambientes locais, pelo } \\
\text { objetivo da economia do conhecimento e que podem ter } \\
\text { poucas opções para reter os alunos para estudar } \\
\text { localmente. }\end{array}$ \\
\hline
\end{tabular}

Fonte: Elaborado pelos autores com base nos dados primários.

\section{CONSIDERAÇÕES}

Este artigo teve como objetivo apontar lacunas empíricas e teóricas acerca das F\&A no setor do ensino superior à luz da Teoria do Crescimento da Firma de Penrose. Por ser um fenômeno altamente presente na realidade nacional, compreender as tendências teóricas internacionais contribui para que se melhor avalie as ocorrências atuais. Evidenciar a importância da teoria do crescimento da firma nos processos de fusões e aquisições no ensino superior também é parte integrante deste artigo, pois revela a necessidade de se desenvolver mais estudos sobre a forma pela qual esta teoria impacta nestas atividades no Brasil.

Um dos objetivos primordiais das empresas é o crescimento. A maior parte das organizações de todos os portes e setores, tem como a sua expansão um fator motivador. Já a falta do crescimento pode demonstrar sinal de fraqueza e decadência.

A partir do quadro 1 foi possivel verificar que as F\&A afetam diretamente diversas estratégias. A saber, segundo Barrier (2014b) que a reorganização formal depende do alinhamento das condições externas e intraorganizacionais.

Um anúncio de uma fusão envia uma forte mensagem ao mercado. A empresa pode estar tentando integrar canais de distribuição, oportunidades de expansão e racionalização, respondendo a mudanças na globalização e na tecnologia. Mais especificamente ao setor educacional, empresas buscam principalmente nas F\&A, reformas estruturais, busca por economias de escala, escopo, vantagem competitiva, implantação de políticas do valor crítico, educacional, mudanças organizacionais, melhora do desempenho acadêmico, entre outros. Sobretudo, conforme o trabalho de Christensen e Erno-Kjolhede (2011), os entrevistados afirmam que as oportunidades e benefícios da fusão claramente superaram ameaças e desvantagens.

Nos últimos dez anos, apenas no Brasil, mais de duas centenas de fusões e aquisições de instituições de Ensino Superior ocorreram. A contribuição teórica e empírica pretendida com os resultados aqui apresentados é impulsionar futuras pesquisas sobre F\&A no setor do ensino superior à luz da Teoria do Crescimento da Firma, de Penrose (1959) por entender que esta vertente teórica tem aspectos essenciais para o tema acima proposto. É importante entender este fenômeno que também ocorreu em inúmeros países do mundo, considerando a importância que o conhecimento e a formação superior adquiriram nas últimas décadas. Além disso, este artigo também visa demonstrar a importância da produção de artigos quantitativos 
para testar hipóteses relacionadas às fusões e aquisições, principalmente estudos que possam analisar amostras significativas dentro do universo nacional e que utilizem variáveis outras que não apenas aquelas relacionadas às performances financeiras.

\section{REFERÊNCIAS}

ADEDOYIN, A. C.; MILLER, M.; JACKSON, M. S.; DODOR, B.; HALL, K. Faculty experiences of merger and organizational change in a social work program. Journal of Evidence-Informed Social Work, v. 13, n. 1, p. 87$98,2016$.

BARNARD, Z.; MERWE, D. V. Innovative management for organizational sustainability in higher education. International Journal of Sustainability in Higher Education, v. 17, n. 2, p. 208-227, 2016.

BARRIER, J. Merger Mania in Science: Organizational Restructuring and Patterns of Cooperation in an Academic Research Centre. Research in the Sociology of Organizations, v. 42, p. 141-172, $2014 a$.

BARRIER, J. The Impact of Institutional Reforms on the Nature of Universities as Organisations, Research in the Sociology of Organizations, v. 42, p. 19-49, 2014 b.

BATES, L. J.; SANTERRE, R. E. A time series analysis of private college closures and mergers. Review of Industrial Organization, v. 17, n. 3, p. 267-276, 2000.

BECKER, L. R.; BEUKES, L. D.; BOTHA, A.; et al. The impact of university incorporation on college lecturers. Higher Education, v. 48, n. 2, p. 153-172, 2004.

BEER, J.; SMITH, U.; JANSEN, C. "Situated" in a separated campus - students' sense of belonging and academic performance: A case study of the experiences of students during a higher education merger. Education as Change, v. 13, n. 1, p. 167-194, 2009.

BOLING J. R.; MAYO D. T.; HELMS M. M. Complementarity merger as a driver of change and growth in higher education. Journal of Organizational Change Management, v. 30, p. 27-42, 2017.

CAMARGOS, M. A.; COUTINHO, E. S. A teoria da firma e a fundamentação teórica para fusões e aquisições: uma análise de suas interfaces. RAC-Eletrônica, v. 2, n. 2, p. 273-295, 2008.

CARTWRIGHT, S.; TYTHERLEIGH, M.; ROBERTSON, S. Are mergers always stressful? Some evidence from the higher education sector. European Journal of Work and Organizational Psychology, v. 16, n. 4, p. 456478, 2007.

CHRISTENSEN, S. H.; ERNO-KJOLHEDE, E. Academic drift in danish professional engineering education. myth or reality? opportunity or threat? European Journal of Engineering Education, v. 36, n. 3, p. 285-299, 2011.

COASE, R. H. The nature of the firm. Economica, London, v. 4, n. 16, p. 386-405, 1937.

COETZEE, S. A.; MBANZE, C. V. Merging into a Private University: Law Regulating the Transfer of Academic Employees in Swaziland. Journal of Asian and African Studies, v. 49, n. 5, p. 584-596, 2014.

DESCHAMPS, E.; LEE, J. J. Internationalization as Mergers and Acquisitions: Senior International Officers' Entrepreneurial Strategies and Activities in Public Universities. Journal of Studies in International Education, v. 19, n. 2, p. 122-139, 2015 
DOCAMPO, D.; EGRET, D.; CRAM, L. The effect of university mergers on the Shanghai ranking. Scientometrics, v. 104, n. 1, p. 175-191, 2015.

DROWLEY, M. J.; LEWIS, D.; BROOKS, S. Merger in higher education: Learning from experiences. Higher Education Quarterly, v. 67, n. 2, p. 201-214, 2013.

FRØLICH, N.; TRONDAL, J.; CASPERSEN, J.; REYMERT, I. Managing mergers - governancing institutional integration. Tertiary Education and Management, v. 22, n. 3, p. 231-248, 2016.

GLASS, J. C.; MCCALLION, G.; MCKILLOP, D. G.; RASARATNAM, S.; STRINGER, K. S. Implications of variant efficiency measures for policy evaluations in UK higher education. Socio-Economic Planning Sciences, v. 40, n. 2 , p. $119-142,2006$.

GLEIBS, I. H.; MUMMENDEY, A.; NOACK, P. Predictors of Change in Postmerger Identification During a Merger Process: A Longitudinal Study. Journal of Personality and Social Psychology, v. 95, n. 5, p. 10951112, 2008.

GLEIBS, I. H.; TÄUBER, S.; TENDAYIVIKI, G.; GIESSNER, S. R. When what we get is not what we want: The role of implemented versus desired merger patterns in support for mergers. Social Psychology, v. 44, n. 3, p. 177-190, 2013.

HALL, M.; SYMES, A. South African higher education in the first decade of democracy: From cooperative governance to conditional autonomy. Studies in Higher Education, v. 30, n. 2, p. 199-212, 2005.

HARMAN, K. The research training experiences of doctoral students related to Australian Cooperative Research Centres. Higher Education, v. 3/4, n. 44, p. 469-492, 2002 a.

HARMAN, K. Merging divergent campus cultures into coherent educational communities: Challenges for higher education leaders. Higher Education, v. 44, n. 1, p. 91-114, 2002 b.

HARMAN, G.; HARMAN, K. Institutional mergers in higher education: Lessons from international experience. Tertiary Education and Management, v. 9, n. 1, p. 29-44, 2003.

HARMAN, G.; HARMAN, K. Strategic mergers of strong institutions to enhance competitive advantage. Higher Education Policy, v. 21, n. 1, p. 99-121, 2008.

HARMAN, K.; MEEK, V. Introduction to special issue: "Merger revisited: international perspectives on mergers in higher education". Higher Education, v. 44, p. 1-4, 2002.

HAY, D.; FOURIE, M. Preparing the way for mergers in South African higher and further education institutions: an investigation into staff perceptions. Higher Education, v. 1, n. 44, p. 115-131, 2002.

HIDALGO-HIDALGO, M.; VALERA, G. University merging process: A guideline proposal for excellenceenhancing. B.E. Journal of Economic Analysis and Policy, v. 16, n. 3, p. 1359-1386, 2016.

HOPER. Análise setorial do ensino superior privado - 2014. Foz do Iguaçu: Hoper, 2014.

JENSEN, M. C.; MECKLING, W. H. Theory of the firm: managerial behavior agency, costs and ownership structure. Journal of Financial Economics, v. 3, n. 4, p. 305-360, 1976.

JOHNES, J. Efficiency and mergers in english higher education 1996/97 to 2008/9: Parametric and nonparametric estimation of the multi-input multi-output distance function. The Manchester School, v. 82, n. 4, p. $465-487,2014$.

JOHNES, J.; YU, L. Measuring the research performance of Chinese higher education institutions using data envelopment analysis. China Economic Review, v. 19, n. 4, p. 679-696, 2008. 
KARODIA, A. M.; SHAIKH, A.; SONI, D. The South African Universities Post - Merger Mess: Problems and Challenges of Transformation. Mediterranean Journal of Social Sciences, v. 6, n. 3, p. 2039-9340, 2015.

KYVIK, S. The merger of non-university colleges in Norway. Higher Education, v. 44, n. 1, p. 53-72, 2002.

KYVIK, S.; STENSAKER, B. Factors Affecting the Decision to Merge: The case of strategic mergers in Norwegian higher education. Tertiary Education and Management, v. 19, n. 4, p. 323-337, 2013.

MABOKELA, R. O.; WEI, N. Lingering effects of the past on a University merger process in post-apartheid South Africa. International Education Journal, v. 8, n. 1, p. 160-171, 2007.

MOKHUBA, D. K.; GOVENDER, K. The merger of historically disadvantaged tertiary institutions in South Africa: A case study of the University of Limpopo. Cogent Business \& Management, v. 20, n. 1, p. 1-12, 2016.

NGCAMU, B. S. Conceptualizing transformation in the post-merger and incorporation environment era: A case of the Durban University of Technology, International Journal of Sociology and Social Policy, v. 36, n. 5/6, p. 270-288, 2016.

NGCAMU, B. S.; AFRICA, S.; TEFERRA, D.; AFRICA, S. Leadership and transformation in a South African university. Problems and Perspectives in Management, v. 13, n. 1, p. 208-216, 2015.

NGCAMU, B. S.; TEFERRA, D. How Well Do University Staff Understand Transformation? A Case of a Merged South African University. International Journal of Science Education, v. 8, n. 2, p. 305-312, 2015.

PATTERSON G. Findings on economies of scale in higher education: implications for strategies of merger and aliance. Tertiary Education and Management v. 6, p. 259-269, 2000.

PAVLIK, L. Transformation in Hungary's Higher Education System. Public Finance Quarterly, v. 61, n. 2, p. $224-243,2016$.

PENROSE, E. T. The Theory of the Growth of the Firm. New York: John Wiley, 1959.

PICK, D. Framing and frame shifting in a higher education merger. Tertiary Education and Management, v. 9, n. 4 , p. 299-316, 2003.

PUUSA, A.; KEKÄLE, J. Feelings over facts - a university merger brings organisational identity to the forefront. Journal of Higher Education Policy and Management, v. 37, n. 4, p. 432-446, 2015.

PUUSA, A.; KEKÄLE, J. Commitment in the Context of a Merger. Tertiary Education and Management, v. 19, n. 3, p. 205-218, 2013.

RIPOLL-SOLER, C.; MOLINA, M. M. University mergers: Content analysis of their typologies and the factors that can determine their success or failure a literature review. Revista de Educación, n. EXTRA, p. 313-337, 2013 .

RIPOLL-SOLER, C.; MOLINA, M. M. Are mergers a win-win strategic model? A content analysis of interinstitutional collaboration between higher education institutions. Tertiary Education and Management, $v$. 20, n. 1, p. $44-56,2014$.

ROSS, S A. et al. Administração Financeira. 10. ed. São Paulo: AMGH, 2015.

SAARTI, J.; JUNTUNEN, A. The benefits of a quality management system. Library Management, v. 32, n. 3, p. 183-190, 2011.

SEHOOLE, M. T. C. The politics of mergers in higher education in South Africa. Higher Education, v. 50, n. 1, p. 159-179, 2005. 
SLADE, C. P.; RIBANDO, S. J.; FORTNER, C. K. Faculty research following merger: a job stress and social identity theory perspective. Scientometrics, v. 107, n. 1, p. 71-89, 2016.

TIGHT, M. Institutional churn: Institutional change in United Kingdom higher education. Journal of Higher Education Policy and Management, v. 35, n. 1, p. 11-20, 2013.

URSIN, J.; AITTOLA, H.; HENDERSON, C.; VÄLIMAA, J. Is education getting lost in university mergers? Tertiary Education and Management, v. 16, n. 4, p. 327-340, 2010.

VARTIAINEN, P. Campus-based tensions in the structural development of a newly merged university: the case of the University of Eastern Finland. Tertiary Education and Management, v. 23, n. 1, p. 53-68, 2017.

WALSH, J. "The problem of Trinity College Dublin": a historical perspective on rationalisation in higher education in Ireland. Irish Educational Studies, v. 33, n. 1, p. 5-19, 2014.

WAN, Y.; PETERSON, M. W. A case study of a merger in Chinese higher education: The motives, processes, and outcomes. International Journal of Educational Development, v. 27, n. 6, p. 683-696, 2007.

YLIJOKI, O. H. University Under Structural Reform: A Micro-Level Perspective. Minerva, v. 52, n. 1, p. 55-75, 2014 .

ZEEMAN, N.; BENNEWORTH, P. Globalisation, mergers and 'inadvertent multi-campus universities': reflections from Wales. Tertiary Education and Management, v. 23, n. 1, p. 41-52, 2017. 